\title{
Inflexões epistemológicas: a Etnopsiquiatria
}

\author{
Lucienne Martins-Borges, (D) I, II $\star$ Mariá Boeira Lodetti, (DD II Márcio Jibrin, (D) I Jean-Bernard Pocreau (D) II \\ ${ }^{I}$ Universidade Federal de Santa Catarina, Florianópolis, SC, Brasil \\ "I Université Laval, Québec, Canadá
}

\begin{abstract}
Resumo
A Etnopsiquiatria, fundada por Geroges Devereux, surge enquanto possibilidade de refletir o encontro intercultural e seus desdobramentos quanto à compreensão do adoecimento psíquico e à coerência entre terapêuticas e etiologias deste adoecimento. A síntese teórica que levou à criação da disciplina emerge de inflexões baseadas principalmente nos estudos de Freud, Malinowski, Róheim e de contribuições posteriores de Lévi-Strauss e Bastide. Tobie Nathan é responsável pela criação do modelo de intervenção que coloca em prática a aplicação da teoria. Este artigo visa apresentar o histórico dos princípios precursores que levaram Devereux à síntese teórica e à criação da Etnopsiquiatria e expor as considerações da experiência adquirida na aplicação da disciplina pelo modelo proposto por Nathan e multiplicado por outros clínicos. Do postulado da universalidade do psiquismo que se constitui na particularidade da cultura, compreende-se uma relação intrínseca existente entre cultura e psiquismo, em que a cultura utiliza-se dos mesmos elementos, processos e mecanismos de defesa do psiquismo. Adotando o duplo discursoPsicanálise e Etnologia - a disciplina define-se complementarista. A Etnopsiquiatria surge enquanto possibilidade diante da lacuna que a lógica da psiquiatria ocidental deixou para explicar o adoecimento psíquico e seu modelo de intervenção viabiliza o posicionamento de aprendiz no encontro intercultural.
\end{abstract}

Palavras-chave: Etnopsiquiatria; cultura; psiquismo.

\section{Epistemological inflexions: the etnopsychiatry}

\begin{abstract}
The Ethnopsychiatry founded by Geroges Devereux emerged as a possibility to reflectabout the intercultural encounter and its unfolding regarding the understanding of psychological disorders and the coherence between therapeutics and etiologies of this psychopathological states. The theoretical thesis that led to the creation of the discipline arises from inflections based mainly on the studies from Freud's, Malinowski's and Rheheim's theory, along with later contributions by Lévi-Strauss and Bastide. Tobie Nathan is later credited for creating an intervention clinical model and a practice. This article aims to present the history of the precursory principles that led Devereux to the theoretical thesis and the resulting creation of Ethnopsychiatry and also to make considerations about the experience acquired in the application of the model of practice proposed by Nathan and multiplied by other clinicians. From the postulate of the universality of the psychological structure which is particularily constituted by culture, the discipline comprises an intrinsic relation existing between culture and the psychic functioning, in which culture uses the same elements, processes and defense mechanisms as the psyche. Adopting the double discourse method - Psychoanalysis and Ethnology -the discipline defines itself as complementary. Ethnopsychiatry emerges as a possibility to look at the gap that Western psychiatry left to explain psychological disorders, as well as to show how its model of intervention advocates to position the discipline as an apprentice in the intercultural encounter.
\end{abstract}

Keywords: Ethnopsychiatry; culture; psyche.

\section{Introdução}

O que é necessário para pensar o encontro intercultural, sobretudo aquele encontro entre duas pessoas com origens culturais organizadas em torno de lógicas distintas? Como criar um encontro possível entre tais lógicas que permita a compreensão do mal-estar do outro e igualmente a identificação dos recursos culturais para as direções do tratamento? A Etnopsiquiatria emerge como maneira de pensar o sofrimento do outro e de estar em relação com ele (POCREAU, 2013). Nesse sentido, dissertar acerca da teoria da Etnopsiquiatria formulada por Georges Devereux (1951) implica evocar considerações daqueles que a utilizam na sua dimensão metodológica e intervencionista, tal qual foi sustentada por Tobie Nathan (1986).

A Etnopsiquiatria é uma disciplina que surge enquanto possibilidade de abordar teorias e processos terapêuticos referentes à saúde mental de populações oriundas de

\footnotetext{
^Endereço para correspondência: Universidade Federal de Santa Catarina, Departamento de Psicologia. Centro de Filosofia e Ciências Humanas. Campus Universitário Trindade -Florianópolis, SC - Brasil. CEP: 88040970. E-mails: lucienne.borges@ufsc.br, maria.boeira-lodetti.1@ulaval.ca, marciojibrin@ gmail.com, jean-Bernard.Pocreau@psy.ulaval.ca

Os dados completos dos autores encontram-se ao final do artigo.
}

culturas não-ocidentais com o objetivo de oferecer um arcabouço teórico e prático que ajude a responder aos questionamentos supracitados (PIERZO; LEGAULT, 2001). Nessa perspectiva, a Etnopsiquiatria origina-se das seguintes questões: Qual a compreensão que grupos sociais não-ocidentais atribuem ao adoecimento psíquico? Há coerência entre as terapêuticas prescritas e as etiologias do respectivo adoecimento? (LAPLANTINE, 1998). Diante da parcialidade de um único saber - evidenciada por Devereux (1985) - a verdadeira Etnopsiquiatria não é interdisciplinar, mas sim pluridisciplinar, tendo em vista que faz dupla análise de um mesmo fato: por um lado com os referenciais da etnologia; por outro, com os da psicanálise. Tal pluridisciplinaridade - compreendida não no sentido de fusão nem no de simultaneidade - é fruto da relação complementar entre dois sistemas explicativos distintos e não exclusivos: o método do duplo discurso. A aliança teórica e metodológica entre estes saberes edifica a Etnopsiquiatria e reflete o olhar que Devereux lançava sobre a constituição subjetiva do homem, como efeito do enlace entre o cultural e o psíquico. Para poder pensar a aplicabilidade do du- 
plo discurso parte-se do principal postulado da Etnopsiquiatria: o da universalidade psíquica na singularidade da cultura. Diante da correspondência entre psíquico e cultural e dos desafios inerentes à alteridade, destaca-se a necessidade de modificação das formas de pensar e de fazer relativas ao tratamento terapêutico (MORO, 1992).

A justificativa do duplo discurso surge da concepção de que a cultura é como a face externa dos processos psíquicos. Do âmago do psiquismo individual às conquistas civilizatórias mais elevadas, a cultura é como a pele que reveste e contém o sujeito do nascimento até a morte. Um reservatório de possibilidades da humanidade, a cultura é o que torna o real suportável, na medida em que lança no simbólico a experiência ainda sem representação. $\mathrm{Na}$ qualidade de suas funções ela pode evitar a confusão e a perplexidade ao carregar consigo muitas das explicações e predições acerca dos eventos da vida e das possibilidades terapêuticas em caso de adoecimento (LAPLANTINE, 1998; MARTINS-BORGES; POCREAU, 2009).

Assim, este artigo visa apresentar o histórico dos princípios precursores que levaram Georges Devereux à síntese teórica e à criação da respectiva disciplina; busca ainda expor as considerações da experiência adquirida na aplicação da disciplina por meio da metodologia interventiva proposta por Tobie Nathan (França) - e multiplicada por outros clínicos, com destaque para Marie-Rose Moro (França), Jean-Bernard Pocreau e Lucienne Martins-Borges (Québec, Canadá) (LECOMTE; JAMA; LEGAULT, 2006). Para tanto, inicia-se com o breve resgate do advento do conceito científico de cultura para as Ciências Humanas e os seus desdobramentos nas teorias psicodinâmicas, destacando a indissociabilidade entre os processos culturais e psíquicos na organização e expressão do sofrimento humano. Por fim, sustenta-se a posição epistemológica e o direcionamento metodológico clínico da Etnopsiquiatria.

\section{Histórico}

O conceito de cultura apresenta grande amplitude e complexidade dada a sua vasta utilização em diversas áreas do conhecimento. Não cabe uma análise exaustiva da evolução do conceito e nuances de uso, e sim a transmissão de elementos suficientes para compreensão do papel da cultura para a formulação da Etnopsiquiatria. Edward Burnett Tylor (1832-1917), antropólogo britânico e representante do pensamento evolucionista, apresenta em sua obra Cultura Primitiva (1871), o primeiro conceito científico de cultura, o que lhe concede o título entre os seus pares de pai do conceito moderno de cultura. Para Tylor (1871, p. 1 apud CUCHE, 1999, p. 35), a cultura é compreendida como um "conjunto complexo que inclui o conhecimento, as crenças, a arte, a moral, o direito, os costumes e as outras capacidades ou hábitos adquiridos pelo homem enquanto membro da sociedade". Cabe salientar que essa conceituação se situa dentro de uma perspectiva evolucionista de metodologia comparativista; o que permite, dentro desse paradigma, pensar a cultura como experiência única e universal e, portanto, categorizada em diferentes níveis de desenvolvimento. Ao subordinar a cultura à uma noção linear do desenvolvimento da história, determinadas sociedades passam a ser nomeadas de primitivas, selvagens ou mesmo bárbaras, em oposição àquelas ditas civilizadas. Essa postura de observar outras culturas em função da sua própria, tomando-a como padrão para valorizar e hierarquizar as restantes, denomina-se etnocentrismo (BOAS, 2004).

Franz Uri Boas (1858-1942), antropólogo alemão incomodado com os limites científicos do argumento biológico para compreender os costumes sociais, e da plasticidade do conceito de raça, aponta que não há sentido em adotar uma postura metodológica comparativista em relação às diferentes culturas. Em sua perspectiva, particularista, é um equívoco falar em uma única cultura humana, pois diante de toda a pluralidade verificada nas expedições de observação, estaríamos diante de inúmeras culturas. Elas até podem ter semelhanças e mesmo equivalências entre si, mas compará-las seria improdutivo, pois uma cultura só pode ser apreendida em seu particularismo histórico. O relativismo cultural pontuado por Boas não invalida o conceito proposto por Tylor, mas a sua forma de utilização. O que Boas critica duramente é a postura etnocêntrica dos pesquisadores ao se defrontarem com uma organização cultural estranha a sua e, por conseguinte, subordiná-la à sua própria concepção (CUCHE, 1999).

Em uma perspectiva psicodinâmica compreende-se a cultura como uma realidade compartilhada, uma herança social, e não genética, transmitida pela educação formal e informal. É um sistema dinâmico, em constante mutação, devido à influência de fatores internos e/ou externos que possibilitam adequação às experiências da vida. Ou, ainda, um sistema de símbolos e de significados próprios de um grupo, cujos diferentes elementos relacionam-se entre si, de forma coerente, e estão em constante interação (GUERRAOUI; PIRLOT, 2011). Portanto, seja sob a ótica antropológica, seja sob a ótica psicodinâmica, a cultura configura-se como elemento organizador do social. Ao mesmo tempo que fornece as possíveis direções para a existência, contém todo o tesouro de produções de um grupo.

Para compreender os elementos utilizados por Devereux para a síntese da sua teoria baseada no complementarismo, cabe rever alguns nomes que marcaram a história, a começar por Freud (1856-1939). O diálogo entre Etnologia e Psicanálise inicia-se quando da publicação de Totem e Tabu (FREUD, 1912-1913/1974), texto por meio do qual Freud pretendia apontar para a universalidade do conceito de complexo de Édipo pela descrição dos significados de totem e tabu em culturas da "psicologia dos povos". Dessa forma, Freud defende nesse texto a premissa de que, assim como ocorre nas sociedades ocidentais, as organizações nas sociedades "primitivas" também se davam pela proibição implícita do incesto e do assassinato do pai. Para tanto, ele descreve os modos como isso ocorria nesses grupos culturais, baseando-se, para criar suas hipóteses teóricas, numa compreensão filogenética darwinista e nos elementos culturais oferecidos por tais sociedades "primitivas" (FERMI, 2008; ROUDINESCO; PLON, 1998). Em críticas à proposta de diálogo entre a Psicanálise e a Etnologia teorizada por Freud em Totem e Tabu, o antropólogo inglês Malinowski 
(1884-1942) defendia a ideia de que uma produção científica somente pode ser reconhecida quando se utiliza um método baseado em fatos que podem ser observados diretamente pelo pesquisador e que podem ser descritos de modo exato (ROUDINESCO; PLON, 1998). Dado que Freud não teve acesso direto aqui, por observação, às culturas utilizadas para a demonstração de sua teoria, Malinowski sustentava sua discordância de que existia um complexo de Édipo universal, comum a todas as culturas, uma vez que a diversificação das funções desempenhadas pelos indivíduos pertencentes aos grupos das sociedades primitivas não correspondia às configurações socioculturais europeias já observadas (FERMI, 2008).

Com o objetivo de responder às críticas feitas por Malinowski e de reunir elementos que baseassem a teoria da universalidade do complexo de Édipo, alguns teóricos da psicanálise - Freud, Ferenczi, Wilma Kovacs e Marie Bonaparte - propõem que alguém com formação em Psicanálise e Etnologia (Etnopsicanálise) vá a campo coletar dados por observação direta e, com as informações colhidas, elaborar um estudo do que observou, uma vez que, dada a sua formação, poderia ser a pessoa mais competente para um diálogo entre as disciplinas (FERMI, 2008). Nesse contexto, Geza Roheim (1891-1953) lança-se em viagem exploratória em 1928. Roheim nasceu em 1891, em Budapeste; descendente de judeus, era filho único de uma família de comerciantes húngaros. Desde pequeno era muito curioso por obras literárias. Aos 12 anos, tinha uma conta aberta em uma das mais importantes livrarias de Budapeste, e, aos 19 anos, apresentou seu primeiro trabalho, sobre a mitologia da lua, para a Sociedade Etnográfica Húngara. Escreveu trabalhos sobre o folclore húngaro e era considerado bastante ligado às expressões pela arte que envolviam sua cultura de origem (ROUDINESCO; PLON, 1998). Com passagem por Yêmen e Djibouti, ele permaneceu durante nove meses em um grupo social da ilha de Normanby. Utilizando elementos obtidos em sua experiência de campo, Roheim elabora uma teoria segundo a qual cada cultura tem seu trauma infantil específico. Nesse sentido, Roheim (apud FERMI, 2008, p. 63, tradução nossa) afirma que

se fosse possível ter um conhecimento psicanalítico aprofundado de todas as culturas, o que existiria de semelhante entre elas é uma situação infantil específica, de angústia infantil ou tendência libidinal que possuem uma função determinada em cada cultura.

Pela noção de trauma infantil singular a cada cultura, Roheim sustentava a premissa de que não há discurso absoluto que corresponda à leitura única do normal e do patológico, uma vez que cada sociedade tem um sofrimento que é próprio dela (FERMI, 2008). A teoria da universalidade do trauma infantil, que ocorre na singularidade de cada cultura, tornou-se, mais tarde, essencial para a constituição da Etnopsiquiatria, visto que a disciplina utiliza a cultura como dimensão norteadora para a compreensão da constituição do sujeito e, por conseguinte, para a compreensão de seu sofrimento psíquico (POCREAU; MARTINS-BORGES, 2013).
Posterior a Roheim, Georges Devereux (1908-1985), ou Gyorgy Dobo - seu nome de nascença -, distingue-se na história como quem unificou a teoria elaborada por psicanalistas, antropólogos e etnopsicanalistas que o precederam e a sua experiência clínica como psicanalista e antropólogo, tornando-se o fundador da Etnopsiquiatria. Nascido no território atualmente reconhecido como a Romênia, Devereux, judeu, imigrante, era filho de mãe alemã e pai húngaro. Em 1947, no contexto de pós-guerra no território dos Estados Unidos, Devereux tem seu primeiro e único paciente, Jimmy Picard, um indígena norte-americano do grupo Blackfoot. Naquele momento, Devereux, já colocava em questão a compreensão única do normal e do patológico, baseando-se na hipótese de que o sintoma é codificado culturalmente. Com seu paciente, Devereux coloca em prática a posição daquele que não sabe, daquele que vai apreender o mundo do outro e com ele compreender os significados que estruturam o seu universo simbólico-cultural. Essa experiência e suas considerações sobre ela são base para o que mais tarde se formaliza como a Etnopsiquiatria.

Destaca-se que nessa época os especialistas norte-americanos tinham grande reconhecimento graças aos tratamentos que ministravam aos soldados que retornavam ao país com sintomas pós-traumáticos. Devereux foi enviado ao encontro de Jimmy Picard devido à ineficácia dos tratamentos até então ofertados a este paciente (NATHAN, 2014). Devereux estudou em Paris com Mauss (1872-1950), etnólogo francês responsável pela fundação do Instituto de Etnologia e pesquisador de temáticas como a relação entre a religião e o sagrado; realizou seu doutorado em Antropologia nos Estados Unidos, em 1961, quando pesquisou sobre os Mohave, povos nativos norte-americanos (ROUDINESCO; PLON, 1998) e "demonstrou que as teorias e as práticas referentes à angústia e os adoecimentos próprios aos universos culturais não-ocidentais são sensatas, coerentes, lógicas e eficazes" (GRANDSARD, 2009, p. 47). Em 1963, Devereux retorna à França, a convite de Claude Lévi-Strauss (19082009) e Roger Bastide (1898-1974), com o qual estudou; começa a lecionar na École Pratique des Hautes Études e, em 1970, funda a Etnopsiquiatria.

Na tese de Devereux, percebe-se ainda a influência da antropologia estrutural de Claude Lévi-Strauss, a qual busca as "invariantes" dos materiais culturais, ou seja, aqueles princípios duradouros e indispensáveis para vida em sociedade. Para Lévi-Strauss, tendo em vista a unidade do psiquismo, os particulares de uma cultura não podem ser compreendidos sem referência às "categorias e estruturas inconscientes do espírito humano" (CUCHE, 1999, p. 95). No ensaio Eficácia Simbólica, o autor descreve um ritual xamânico de cura para a resolução de um parto difícil e chega à conclusão de que, independentemente da mitologia xamã corresponder a uma lógica e realidade objetiva ocidental, ela funciona. Funciona, pois, a parturiente, submetida ao ritual e à sociedade da qual ela é membro acreditam na mitologia e na figura do xamã, membro da mesma cultura. Esse exemplo ilustra como o universo simbólico do homem organiza-se pela cultura, 
e mostra que quando existe coerência entre o internalizado pelo sujeito e a intervenção à qual ele é submetido, a eficácia se comprova (LÉVI-STRAUSS, 2012). O que evidencia o papel do antropólogo francês nas referências da Etnopsiquiatria no que concerne às pesquisas antropológicas acerca da eficácia de tratamentos em culturas não-ocidentais (MORO, 1992)

Salienta-se que o nome Etnopsiquiatria não foi criado por Devereux, mas tomado emprestado de Louis Mars (1906-2000), psiquiatra haitiano. A escolha de Devereux no emprego dessa expressão deve-se ao fato do termo Etnopsiquiatria referir-se à relação entre sintoma e cultura, assim como, à eficácia das terapias tradicionais (ou seja, não-ocidentais) tanto quanto à eficácia - já reconhecida - das terapias ocidentais. Assim, o uso do termo corresponde à tentativa de uma geopolítica da psicopatologia, visto que os saberes não-ocidentais eram igualmente considerados um saber, um conhecimento, independentemente dos critérios científicos ocidentais que pejorativamente os classificavam como "crenças". Esses saberes tradicionais são conhecidos por integrantes às próprias culturas tradicionais/não-ocidentais, pessoas que ocupam um lugar de cuidado no grupo cultural e que dominam diferentes técnicas de tratamento - as quais podem ser ensinadas e mesmo teorizadas, experimentadas, adaptadas e utilizadas em dispositivos clínicos pertencentes a outros contextos (NATHAN, 2005).

Sucessor de Devereux e responsável pela criação do modelo de intervenção clínica da Etnopsiquiatria, Tobie Nathan realiza em 1979, no Hospital Avicenne (Bobygny), França, seu primeiro atendimento em Etnopsiquiatria. A principal diferença entre a clínica psicanalítica tradicional e a Etnopsiquiatria é o lugar que esta concede à especificidade cultural enquanto base teórico-metodológica. Se a cultura constitui uma parte do sujeito de "ser/ estar no mundo" (NATHAN, 1993), é indispensável a recorrência a ela para a compreensão da expressão do sofrimento psíquico (FERRADJI, 2010). O ponto essencial da Etnopsiquiatria é que não existe homem sem cultura. Em uma metáfora, Nathan (1993) afirma que o homem, assim como possui um fígado, possui uma cultura. Nesse sentido, a Etnopsiquiatria propõe-se a evidenciar as ligações existentes entre o psiquismo e a cultura por meio do argumento de que esta possui papel essencial na constituição do sujeito (MORO, 2001). Há uma equivalência entre ter cultura e ser dotado de psiquismo (NATHAN, 1993). Assim, a teoria da Etnopsiquiatria é baseada no postulado da universalidade psíquica do sujeito, que se dá na singularidade da cultura de origem (LAPLANTINE, 1998)

\section{Cultura e Psiquismo}

A relação entre cultura e psiquismo é tão intrínseca que se pode pensar que em cada indivíduo há dois sistemas redundantes de estrutura homóloga: um de origem interna - o aparelho psíquico; outro de origem externa - a cultura; ambos os sistemas coexistem e têm consequências lógicas importantes na estruturação do sujeito (NATHAN, 1994). Nathan (1986) sustenta que a cultura se utiliza dos mesmos elementos, processos e mecanismos de defesa que o psiquismo; assim, cultura e psiquismo funcionam em dupla, como organizações a que, por meio do conhecimento etnológico e psicológico, se pode ter acesso. Ao encontro disso, "o aparelho psíquico funciona enquanto máquina criadora de ligações, autorregulando-se sobre outra máquina similar, mas de origem externa: a cultura" (NATHAN, 1993, p. 299, tradução nossa); ele atua nos mesmos processos e utiliza-se dos mesmos recursos dos mecanismos de defesa que a cultura faz uso. Dessa forma, fica explícita a justificativa da necessidade de se recorrer ao discurso etnológico e psicanalítico para a compreensão do humano enquanto ser partícipe de uma cultura. Esta é a noção do complementarismo da Etnopsiquiatria, em outras palavras, cultura e psiquismo são estruturalmente coemergentes.

Para esta exposição, pode-se definir cultura como um sistema composto por diferentes elementos que fornecem ao sujeito indicações de como agir e pensar de forma coerente (MORO, 2015). Metaforicamente, é possível relacionar a cultura com uma bússola que orienta o sujeito no curso de sua vida. Ela é composta por elementos significativos, que, dotados de propriedades e de funções - e estruturados de forma dinâmica e autorregulada -, possibilitam a construção de sentido às vivências e eventos da vida (MORO, 2001). Assim, os elementos culturais referem-se, por exemplo, aos valores; às práticas; aos hábitos; às formas de composição familiar; às formas como se organizam e do que são feitos os rituais - de passagem, de alimentação (refeição, de preparo); à lógica de funcionamento e organização de uma sociedade (hierarquias, funções sociais), etc. Referem-se, ainda, os objetos terapêuticos, como orações, cantos, plantas (NATHAN, 1998). Enfim, a cultura, que consiste num conjunto de símbolos que permite ao bebê humanizar-se, representa uma ponte entre o sujeito e o seu ambiente, entre o mundo interno e o mundo externo, proporcionando possibilidades de ser e de fazer, possibilidades de cuidado e de transmissão social por meio dos significados (GUERRAOUI, 2011; MARTINS-BORGES; POCREAU, 2009; MORO, 2001).

Se a cultura significa esse conjunto de produções humanas, compartilhado e transmitido pelas relações, infere-se que ela é implícita e pré-existente à chegada de novos membros a um grupo cultural. Relativamente à constituição psíquica, a cultura expressa-se nas significações dos pais em relação ao bebê antes mesmo da chegada deste ao mundo. Assim, ela é transmitida pelos primeiros investimentos afetivos, pelos primeiros vínculos estabelecidos, estruturando o funcionamento psíquico. A cultura proporciona aos membros de um grupo continência e proteção frente ao real, como se fosse um envelope (MARTINS-BORGES; POCREAU, 2009). Nesse sentido, destaca Grandsard (2009, p. 47), sobre a teoria de Nathan, "este envelope é indispensável para a construção e o equilíbrio psíquico dos indivíduos [...], e consiste em um conjunto de códigos e de práticas que permitem que o mundo seja compreensível para o sujeito e mesmo previsível, de modo a proteger os humanos da perplexidade e do medo". Desse modo, pela compreensão da função da cultura e da sua intrínseca relação com o 
psiquismo, é possível deduzir que a separação do sujeito de seu contexto cultural de origem pode levar à ruptura desse envelope cultural protetor (GRANDSARD, 2009).

Ainda em termos de suas funções, Guerraoui e Pirlot (2011) ressaltam que é a cultura que delimita o que é normal e desviante dentro de determinado contexto. Betts (2013) afirma que todas as culturas têm em seu fundamento aquilo que lhe é de "dentro", ou seja, familiar; e aquilo que lhe é de "fora", ou seja, estranho às lógicas dominantes do contexto. Assim, o autor pontua que a diferença reside no âmago de toda construção cultural. Observa-se que o que é definido como estranho, externo à cultura, tem geralmente representação hostil, é ameaçador e, portanto, deve ser excluído. É justamente nessa diferença vivida na relação com o outro, o não-semelhante, que reside a potência da Etnopsiquiatria, pois, em vez de excluir o estranho e tentar ignorar a alteridade, é no reconhecimento da diferença que está alicerçada a potencialidade de suas investigações e intervenções.

\section{Codificação do Sintoma}

Na compreensão da Etnopsiquiatria, a articulação entre a cultura e o psiquismo é dinâmica e permanente. A cultura serve de base para o funcionamento psíquico; ela possibilita a mediação entre a realidade externa e o mundo psíquico, permitindo ao sujeito dar sentido a esse real e, assim, suportar a existência (MARTINS-BORGES; POCREAU, 2009). O distanciamento do sujeito de seu contexto cultural pode levá-lo a um estado de vulnerabilidade psíquica, uma vez que o equilíbrio possibilitado pela articulação entre a realidade externa e o mundo psíquico é fragilizado pela separação do quadro cultural de referência. Se antes, no contexto cultural de origem, existia uma coerência na decodificação dos significados, diante do afastamento, tal coerência e as estratégias de defesa podem se tornar, naturalmente, menos eficazes. A falta de coerência seria um resultado das diferenças encontradas no novo contexto cultural, em que o local, as pessoas, as lógicas de organização social modificaram-se significativamente. No caso de um afastamento involuntário, por exemplo, isso se acentua mais ainda em razão do caráter forçado do distanciamento cultural e das vivências traumáticas pelas quais passam aqueles que deixam seu contexto de origem. A continuidade e a coesão encontram-se partidas, o que dificulta a esse sujeito de se reconhecer em si mesmo (MARTINS-BORGES, 2017).

A noção do sintoma enquanto uma formação de compromisso do psiquismo com as exigências da realidade faz dele uma produção singular. Assim, a sua expressão está condicionada aos elementos culturais a disposição, uma vez que é a cultura que fornece a materialidade para o sintoma se constituir e ser interpretado. Para Laplantine (1998, p. 73), “os mecanismos psíquicos nada mais são do que a face interna dos processos culturais que podem ser, desse ponto de vista, qualificados de externos". Logo, a escuta do sintoma para a Etnopsiquiatrianão se limita na busca de um quadro diagnóstico - por mais importante que seja o diagnóstico estrutural - mas também na escuta do sintoma como possibilidade de acesso ao so- frimento, à sua etiologia e as possibilidades de tratamento. $\mathrm{Na}$ medida em que a coerência se restabelece, lutos são elaborados e novas ligações são estabelecidas; o que possibilita novos vínculos, diminuição do sofrimento, pertencimento e retomada da continuidade de si.

\section{Considerações finais}

A Etnopsiquiatria é uma disciplina que trata das exceções. Essa prerrogativa de ocupar-se de exceções surge no momento em que abordagens baseadas em lógicas ocidentais da psiquiatria foram insuficientes para construir uma hipótese que pudesse explicar o mal-estar vivido por pessoas originárias de grupos culturais tradicionais. Também, como estes expressavam tal sofrimento e como poderiam, por meio da intervenção terapêutica, reativar seus recursos culturais.

Nesse sentido, para preencher a lacuna que a lógica da psiquiatria ocidental deixou, eis a noção da posição de aprendizagem da Etnopsiquiatria. Aquele que vai tratar assume que não tem todo o conhecimento necessário e se coloca na posição de aprendiz: vai junto com o outro, nesse encontro intercultural, construir uma hipótese coerente culturalmente, uma hipótese que acolha o sujeito, assim como seu sofrimento, quando houver. Nathan (1993) alude a Etnopsiquiatria como o desconhecido que se encontra na fronteira das ciências. Tal afirmação, paralela ao discurso científico enquanto algo ocidental, constitui, por si só, um importante obstáculo epistemológico para a Etnopsiquiatria (NATHAN, 1986), que no rol das ciências ocupa o lugar de aprendiz.

Longe de buscar uma única, a Etnopsiquiatria se apresenta enquanto possibilidade epistemológica de inclusão. A diversidade cultural não deve ser adaptada às teorias e práticas de saúde mental existentes, mas, pelo contrário. É a ciência e as práticas de saúde que devem seguir o rumo dessa diversidade.

\section{Informações sobre os autores:}

\section{Lucienne Martins Borges}

(iD) https://orcid.org/0000-0003-4323-116X

9 http://lattes.cnpq.br/3388192539897247

Professora da École de travail social et de criminologie da Université Laval (Québec, Canadá) e Docente do Programa de Pós-Graduação em Psicologia da Universidade Federal de Santa Catarina (UFSC). Integrante da equipe de pesquisa ÉDIQ Équipe de recherche en partenariat sur la diversité culturelle et 1?immigration dans la région de Québec (Canadá) e colaboradora do Núcleo de Estudos sobre Psicologia, Migrações e Culturas (NEMPsiC/UFSC). Doutora em Psicologia (Ph.D., Université du Québec à Trois-Rivières - Canadá), possui graduação em Psicologia pela Universidade Católica de Goiás (1991), mestrado em Estudos Literários - Université du Quebec, Canadá (1996) e mestrado em Psicologia - Université Laval, Canadá (2000). Cofundadora do Service d'Aide Psychologique Spécialisée aux Immigrants et Réfugiés (Université Laval/CIUSSS-CN, Québec, Canadá). Líder do Grupo de Pesquisa "Psicologia, cultura e saúde mental". Interessa-se principalmente pelos seguintes temas: Psicologia clínica intercultural, Etnopsiquiatria, migrações, refúgio e saúde mental. 
Mariá Boeira Lodetti

(iD) https://orcid.org/0000-0002-2497-2352

(9) http://lattes.cnpq.br/2457084454057566

Possui graduação e mestrado em Psicologia pela Universidade Federal de Santa Catarina (UFSC). Colaboradora do Núcleo de Estudos sobre Psicologia, Migrações e Culturas (NEMPsiC), no qual integra o Grupo de Pesquisa "Psicologia, Cultura e Saúde Mental", nas linhas de pesquisa "Violência e Trauma" e "Migrações, processos psicológicos e saúde mental". Atualmente é doutoranda da École de Travail Social et Criminologie da Université Laval (Québec - Canadá) e membro estudante da Équipe de recherche en partenariat sur la diversité culturelle et l'immigration dans la région de Québec (ÉDIQ).

\section{Márcio Jibrin}

(iD) https://orcid.org/0000-0001-5951-3343

\section{(9) http://lattes.cnpq.br/2537504755403749}

Psicólogo. Mestre em Psicologia da Universidade Federal de Santa Catarina (UFSC). Colaborador do Núcleo de Estudos sobre Psicologia, Migrações e Culturas (NEMPsiC), no qual integra o Grupo de Pesquisa "Psicologia, Cultura e Saúde Mental", nas linhas de pesquisa "Violência e Trauma" e "Migrações, processos psicológicos e saúde mental". Compõe a equipe de terapeutas da Clínica Intercultural, vinculada ao NEMPsiC. Membro do Grupo de Apoio aos Imigrantes e Refugiados em Florianópolis e região - GAIRF. Psicólogo voluntário na Pastoral do Migrante de Florianópolis e supervisor local de estágio na mesma instituição.

\section{Jean-Bernard Pocreau}

\section{(iD) https://orcid.org/0000-0002-0888-5101}

\section{(9) http://lattes.cnpq.br/6634194924578516}

Possui graduação em psychologie pela Université de Rennes I(1968), especialização em Certification à la pratique de la psychothérapie pela C.P.P.Q Montréal(1986) e doutorado em Psicologia pela Université Laval(1977). Atualmente é professor titular da Université Laval, Professor colaborador da Universidade Federal de Santa Catarina e Coordenador responsável da Service d'Aide Psychologique aux Immigrants et Réfugiés. Tem experiência na área de Psicologia.

\section{Contribuições dos autores}

Todos os autores participaram da concepção, análise de resultados, contribuíram efetivamente na realização do artigo e aprovaram a versão final do manuscrito.

\section{Como citar este artigo:}

\section{ABNT}

MARTINS BORGES, Lucienne et al. Inflexões epistemológicas: a Etnopsiquiatria. Fractal: Revista de Psicologia - Dossiê Psicologia e epistemologias contra-hegemônicas, Niterói, v. 31, n. esp., p. 249 255, set. 2019. https://doi.org/10.22409/1984-0292/v31i_esp/29001

\section{APA}

Martins Borges, L., Boeira Lodetti, M., Jibrin, M., Pocreau, J.-B. (2019, set.). Inflexões epistemológicas: a Etnopsiquiatria. Fractal: Revista de Psicologia - Dossiê Psicologia e epistemologias contrahegemônicas, 31(esp.), 249-255. doi: https://doi.org/10.22409/19840292/v31i_esp/29001

\section{Referências}

BETTS, Jaime. Diferença cultural, sofrimentos da identidade e a clínica psicanalítica hoje. SIG Revista de Psicanálise, v. 2, n. 1, p. 85-97, 2013. Disponível em: http://sig.org.br/wp-content/ uploads/2016/04/Num_2_EmPauta2.pdf. Acesso em: 22 jul. 2018.

BOAS, Franz. Antropologia Cultural. CASTRO, Celso (Org). Rio de Janeiro: J. Zahar, 2004.

CUCHE, Denys. A noção de cultura nas ciências sociais. Bauru: EDUSC, 1999.

DEVEREUX, George. Reality and dream: the psychotherapy of a plains indian. New York: International Univ Press, 1951.

DEVEREUX, George. Ethnopsychanalyse complémentariste. Paris: Flammarion, 1985.

FERMI, Patrick. Géza Róheim ou la psychanalyse en voyage. L'Autre, v. 9, n. 1, p. 57-66, 2008. https://doi.org/10.3917/ lautr.025.0057

FERRADJI, Taïeb. Culture et psychopathologie chez les jeunes migrants musulmans installés en France. In: MEKKIBERRADA, Abdelwahed. (Ed.). L'Islam en anthropologie de la santé mentale: théorie, ethnographie et clinique d'un regard alternatif. Münster: Lit Verlag, 2010. p. 135-149.

FREUD, Sigmund. Totem e Tabu (1913). In: SALOMÃO, Jayme (Org.). Obras Psicológicas Completas de Sigmund Freud. Rio de Janeiro: Imago, 1974. v. 13, p. 13-194. Edição Standard Brasileira.

GRANDSARD, Catherine. Enveloppe culturelle et attachement social. Santé Mentale, n. 135, p. 46-49, févr. 2009. Disponible sur: http://www.ethnopsychiatrie.net/CGrandsardEnvelop.htm. Consulté le: 21 juil. 2018

GUERRAOUI, Zohra.; PIRLOT, Gérard. Comprendre et traiter les situations interculturelles. Bruxelas: Groupe de Boeck, 2011.

LAPLANTINE, François. Aprender Etnopsiquiatria. São Paulo: Brasiliense, 1998.

LECOMTE, Yves; JAMA, Sophie; LEGAULT, Gisèle. Présentation: L'ethnopsychiatrie. Santé mentale au Québec, v. 31, n. 2, p. 7-27, 2006.

LÉVI-STRAUSS, Claude. Antropologia Estrutural. São Paulo: Cosac Naify, 2012.

MARTINS-BORGES, Lucienne. Migrações involuntárias e impactos psíquicos: a mediação da cultura. In: PERES, Rodrigo Sanches et al. (Org.). Sujeito contemporâneo, saúde e trabalho: múltiplos olhares. São Carlos: Edufscar, 2017. p. 169-186.

MARTINS-BORGES, Lucienne; POCREAU, Jean-Bernard. A identidade como fator de imunidade psicológica: contribuições da clínica intercultural perante as situações de violência extrema. Psicologia: Teoria e Prática, São Paulo, v. 11, n. 3, p. 224-236, 2009. Disponível em: http://pepsic.bvsalud.org/scielo. php?script=sci_arttext\&pid=S1516-36872009000300016\&lng $=$ pt\&nrm=iso. Acesso em: 13 jun. 2018.

MORO, Marie-Rose. Principes théoriques et méthodologiques de l'ethnopsychiatrie: L'exemple du travail avec les enfants de migrants et leurs familles. Santé mentale au Québec, v. 17, n. 2, p. 71- 98, 1992.

MORO, Marie-Rose. Parents en exil: psychopathologie et migrations. 2. ed. Paris: PUF, 2001. 
MORO, Marie-Rose. Psicoterapia transcultural da migração. Psicologia USP, São Paulo, v. 26, n. 2, p. 186-192, 2015. http:// dx.doi.org/10.1590/0103-6564D20140017

NATHAN, Tobie. La folie des autres : traite d'ethnopsychiatrie clinique. Paris: Bordas, 1986.

NATHAN, Tobie. Fier de n'avoir ni pays, ni amis, quelle sottise c'était. Paris: Dunod, 1993.

NATHAN, Tobie. L'Influence qui guérit. Paris: Odile Jacob, 1994.

NATHAN, Tobie. Georges Devereux et l'ethnopsyquiatrie clinique. Nouvelle Revue d'Ethnopsyquiatrie, n. 35-36, p. 7-18, 1998.

NATHAN, Tobie. Entretien avec Tobie Nathan: questions d'ethnopsychiatrie. Outre-Terre, v. 2, n. 11, p. 575-581, 2005.

NATHAN, Tobie. Comment faire place à l'étranger? L'ethnopsyquiatrie et la loi de l'hospitalité. Conférence de Tobie Nathan le 11 février 2014, à l'Institut des Hautes Études des Communications Sociales (Bruxelles).

PIERZO, Marie-Élizabeth; LEGAULT, Gisèle. L'interface Ethnopsychiatrie et intervention sociale. In : LEGAULT, Gisèle. (Org.). L'Intervention interculturelle. Gaëtan Morin: Boucherville, 2001. p. 286-287.

POCREAU, Jean-Bernard. De l'ethnopsychiatrie à la psychiatrie transculturelle: un parcours sensible à la rencontre de l'autre. In: SEFFAHI, Mohammed (Dir.). Autour de Laplantine: d'une rive à l'autre. Paris: Archives contemporaines, 2013. p. 71-89.

POCREAU, Jean-Bernard; MARTINS-BORGES, Lucienne. La cothérapie en psychologie clinique interculturelle. Santé mentale au Québec, v. 38, n. 1, p. 227-242, 2013. https://doi. org/10.7202/1019194ar

ROUDINESCO, Elisabeth; PLON, Michel. Dicionário de Psicanálise. Rio de Janeiro: J. Zahar Editor, 1998. 